\title{
The Lowest Metallicity Stars in the LMC: Clues from MaGICC Simulations
}

\author{
Chris B. Brook ${ }^{1,4}$, Maider S. Miranda ${ }^{1,2}$, Brad. K. Gibson ${ }^{2}$, Kate Pilkington ${ }^{2}$ and Greg S. Stinson ${ }^{3}$ \\ ${ }^{1}$ Departamento de Física Teórica, Universidad Autónoma de Madrid, E-28049 Madrid, Spain \\ ${ }^{2}$ Jeremiah Horrocks Institute, University of Central Lancashire, Preston, PR1 2HE, UK \\ ${ }^{3}$ Max-Planck-Institut für Astronomie, Königstuhl 17, 69117, Heidelberg, Germany \\ ${ }^{4}$ Email: cbabrook@gmail.com
}

(Received February 15, 2013; Accepted June 14, 2013; Online Publication July 31, 2013)

\begin{abstract}
Using a cosmological hydrodynamical simulation of a galaxy of similar mass to the Large Magellanic Cloud (LMC), we examine the predicted characteristics of its lowest metallicity populations. In particular, we emphasise the spatial distributions of first (Pop III) and second (polluted by only immediate Pop III ancestors) generation stars. We find that primordial composition stars form not only in the central galaxy's progenitor, but also in locally collapsed subhaloes during the early phases of galaxy formation. The lowest metallicity stars in these subhaloes end up in a relatively extended distribution around the host, with these accreted stars possessing present-day galactocentric distances as great as $\sim 40 \mathrm{kpc}$. By contrast, the earliest stars formed within the central galaxy remain in the inner region, where the vast majority of star formation occurs, for the entirety of the simulation. Consequently, the fraction of stars that are from the earliest generation increases strongly with radius.
\end{abstract}

Keywords: galaxies: abundances - galaxies: evolution - methods: numerical

\section{INTRODUCTION}

The baryonic component of the Large Magellanic Cloud (LMC) is dominated by its large off-centre bar and underlying stellar disc $\left(\sim 2.7 \times 10^{9} \mathrm{M}_{\odot}\right)$ van der Marel et al. (2002), in addition to its associated neutral gas component $(\sim 0.5 \times$ $10^{9} \mathrm{M}_{\odot}$ ). The bar is dominated by young stars (Holtzman et al. 1999; Harris \& Zaritsky 2009) and shows a dearth of stars with ages of $\sim 5-12$ Gyr. Photometric surveys of the disc also provide evidence for a comparably quiescent period of star formation at intermediate ages (e.g. SmeckerHane et al. 2002; Harris \& Zaritsky 2009). Beyond the disc region, empirical evidence for the presence of an extended, and old, stellar halo (reaching to at least $\sim 25 \mathrm{kpc}$ ) also now exists (Muñoz et al. 2006).

The distribution of the oldest and the most metal-poor stars in galaxies provides a unique 'near-field' probe of the cold dark matter $(\Lambda \mathrm{CDM})$ hierarchical framework with their relative numbers (compared with the bulk of the metallicity distribution function (MDF) providing insight into the complex interplay between accretion, outflows, star formation efficiencies, and internal mixing processes, e.g., Pilkington et al. 2012b). Indeed, the search for the most metal-poor stars in the Milky Way remains an important goal of contemporary astronomy (e.g. Norris et al. 2013, and references therein). Analogous searches for such first or second generation stars within the LMC are obviously hampered by the latter's distance ( $\sim 50 \mathrm{kpc}$; Gibson 2000). Having said that, neither the stellar component of the bar region (traced by AGB stars; Cole et al. 2005) nor that of the older underlying population (traced by RR Lyrae: Haschke et al. 2012) have thus far shown evidence for the presence of stars with metallicities below $[\mathrm{Fe} / \mathrm{H}] \sim-3 .{ }^{1}$

Beyond the extreme metal-poor tail of stellar abundances within the LMC, there also exists empirical evidence of both an age-metallicity (Holtzman et al. 1999; Cole et al. 2005; Rubele et al. 2011) and age-radius (Piatti \& Geisler 2013) gradient, with essentially no significant radial metallicity gradient (Cioni 2009; Cole et al. 2009; Feast, Abedigamba, \& Whitelock 2010). In consort, such trends support a scenario in which the LMC formed in an 'outside-in' manner (cf. the canonical 'inside-out' scenario usually associated with the Milky Way, e.g., Pilkington et al. 2012a).

\footnotetext{
${ }^{1}$ Although one might not expect to find RR Lyrae stars with $[\mathrm{Fe} / \mathrm{H}]<-3$, as such extremely metal-poor stars may not enter the instability strip (Yoon $\&$ Lee 2002).
} 
Significant progress has been made in relation to predicting the expected distribution of old and metal-poor stars within our galaxy, using Milky Way scale disc simulations. Both White \& Springel (2000) and Diemand, Madau, \& Moore (2005) suggest that the central regions today are the best place to search for the oldest stars. However, the link between the oldest stars, metal-free (Pop III) stars, and extremely metal-poor (pseudo-second generation) stars is not so straightforward.

In Scannapieco et al. (2006) and Brook et al. (2007), we demonstrated that Pop III stars formed over a range that peaked at $z \sim 10$, but continued to form down to $z \sim 5$, and were distributed over a wide range of galactocentric radii at $z=0$. Under the assumption that the Pop III initial mass function (IMF) allowed stars with appropriately low mass to survive until the present day, our prediction was that they would be distributed throughout the galactic halo. On the other hand, the oldest stars formed in haloes that collapsed close to the highest density peak of the final system, and at $z=0$ were located in the central or bulge region of the galaxy. These two studies also predicted similar distributions for second generation stars-i.e., those enriched only by Pop III stars-and showed that the fraction of first and second generation stars compared to the total stellar population was higher in the extended halo, compared with the bulge.

The question now arises; will old and metal-poor stars be distributed in a similar manner in lower mass galaxies, such as the LMC? Hierarchical build-up of mass is (almost) selfsimilar with respect to different mass haloes in a $\Lambda \mathrm{CDM}$ Universe (e.g. Macciò, Dutton, \& van den Bosch 2008). That said, the stellar mass-halo mass relation (Moster et al. 2010; Guo et al. 2010) and the missing satellite problem (Klypin et al. 1999; Moore et al. 1999) suggest that baryon fraction is a strong function of total mass, and that there may be a mass below which dark matter haloes have no baryons. ${ }^{2}$ Should the LMC have an accreted population of stars, given its mass? According to Purcell, Bullock, \& Zentner (2007), the answer would be yes, with an accreted diffuse stellar component of mass $\sim 10^{7} \mathrm{M}_{\odot}$. Will the accretion events responsible for this component also bring in first/second generation stars and, if so, how will they be distributed in an LMC-like system? In this study, we examine a fully cosmological simulation of a dwarf irregular which has a comparable stellar mass to that of the LMC, in order to answer these questions.

A brief overview of the code and the underlying physics associated with the simulation employed here is provided in Section 2. We then (Section 3) examine the resolution of the simulations and relation between the sites of first star formation in our simulations to what is found in specialised high resolution simulations focussing on early star formation. The primary results (Section 4) and conclusions (Section 5) are drawn thereafter.

\footnotetext{
${ }^{2}$ The existence of baryons in low mass haloes may be a combination of collapse time and mass, rather than mass alone, with haloes that collapse later less likely to house baryons (see e.g. Bullock, Kravtsov, \& Weinberg 2000).
}

\section{THE SIMULATION}

We make use of initial conditions drawn from the MacMaster Unbiased Galaxy Simulations (MUGS; Stinson et al. 2010), but employ the feedback prescription outlined by the Making Galaxies in a Cosmological Context collaboration (MaGICC; Brook et al. 2012a). We inject $10^{51}$ erg per supernova in the form of thermal energy into the surrounding interstellar medium (ISM), using the blastwave formalism of Stinson et al. (2006). ${ }^{3}$ Cooling is disabled for gas particles situated within a blast region of size $\sim 100 \mathrm{pc}$, for a time period of order $10 \mathrm{Myr}^{4}{ }^{4}$ We adopt a Chabrier (2003) IMF, and include the effects of radiation energy from massive stars in the $\sim 4 \mathrm{Myr}$ prior to the appearance of a stellar particle's first Type II supernova. The efficiency with which this latter energy couples to the ISM, though, is $<1 \%$. Star formation is restricted to regions which are both sufficiently cool $(<15000 \mathrm{~K})$ and dense $\left(>9.3 \mathrm{~cm}^{-3}\right)$.

The initial gas (dark matter) particle mass of the simulation is $2.4 \times 10^{4} \mathrm{M}_{\odot}\left(1.7 \times 10^{5} \mathrm{M}_{\odot}\right)$, while stars are formed with mass $7.9 \times 10^{3} \mathrm{M}_{\odot}$. The gravitational softening length is $155 \mathrm{pc}$ for all particles.

The simulation employed here-our LMC analogue-was utilised in our earlier work to study its global photometric and kinematic characteristics ${ }^{5}$ (Brook et al. 2012a), the chemical properties of its stellar disc (Brook et al. 2012a), and the skewness, kurtosis, and higher order moments of its $\mathrm{MDF}^{6}$ (Pilkington et al. 2012b). Its stellar mass $\left(4.2 \times 10^{9} \mathrm{M}_{\odot}\right)$ and rotation velocity ( $\sim 85 \mathrm{~km} \mathrm{~s}^{-1}$ at 2.2 disc scale lengths) mean that it is somewhat more massive than the LMC and, as such, all comparisons are made with this caveat in mind.

The simulation, along with its companions within the MaGICC suite, is consistent with the empirical stellar masshalo mass relation (Brook et al. 2012a; Stinson et al. 2013) as derived by halo-matching studies (Moster et al. 2010; Guo et al. 2010), as well as the evolution of such relations (Moster, Naab, \& White 2013; Stinson et al. 2013); in addition, they match a wide range of galaxy scaling relations (Brook et al. 2012a). This gives a degree of confidence in the history of the build-up of the baryonic mass within the dark matter-dominated growth of the galaxy mass, and in particular that there are reasonable constraints on the accretion of stellar systems when mergers occur. Further, our simulations include strong outflows (Brook et al. 2011), particularly in dwarfs, and reproduce the extended metal enrichment of the circumgalactic media of intermediate mass galaxies (Stinson et al. 2012), and possess temporally invariant metallicity gradients (Pilkington et al. 2012b; Gibson et al. 2013).

\footnotetext{
${ }^{3}$ The radius over which the energy is deposited corresponds to radius at which the interior pressure in the remnant is reduced to that of the pressure of the ambient ISM, i.e., $\mathrm{R}_{\text {merge }}$, using the terminology of Gibson (1994).

${ }^{4}$ This is the timescale for the remnant's interior to cool to $\sim 10^{4} \mathrm{~K}$, below which radiative losses are minimal; this corresponds roughly to a timescale $\sim 200 \times$ that associated with the formation of the thin dense shell, subsequent to the radiative cooling of the shocked material (the latter occurs near the end of the Sedov-Taylor phase) (Gibson 1994).

${ }^{5}$ Where it was referred to by the label SG3.

${ }^{6}$ Where it was referred to by the label $11 \mathrm{mChab}$.
} 
Extended stellar haloes in low mass galaxies may form from processes other than accretion. Stinson et al. (2009) show that elevated early star formation activity combined with supernova feedback can produce an extended stellar distribution in the absence of accretion, while Zolotov et al. (2009) and House et al. (2011) show that stars formed in situ can also contribute to stellar haloes. These processes are self-consistently included in our simulations, and are thus accounted for in our analysis of the distribution of low metallicity populations. Further, in this study, we analyse an isolated galaxy formation simulation that is the closest analogue to the LMC in terms of stellar mass of those formed in the MaGICC program. Thus, an assumption of this paper is that the early stars in the LMC are not enriched by outflows from the progenitor of the Milky Way. Given the analysis of Scannapieco et al. (2006), who showed that even in models with extreme outflows, lower mass objects which collapse away from the central galaxy are formed from primordial gas, this would appear to be a reasonable assumption, particularly if the LMC is truly on its first orbit around the Milky Way (Besla et al. 2007).

Finally, we note that our simulation does not incorporate an event which results in an analogue of the significant bar seen in the LMC. Instead, our study aims to explore the formation and evolution of the older populations and underlying disc stars. While not meaning to be a one-to-one 'clone' of the LMC, we will see that our model can provide some broad insights into the lowest metallicity populations of stars expected of galaxies of this mass.

\section{EARLIEST STAR FORMING SITES}

The nature of the sites of first star formation remains uncertain and the subject of significant research. Early analytic models found variously that the earliest stars form within haloes of mass between $\sim 5 \times 10^{4} \mathrm{M}_{\odot}$, and $\sim 5 \times 10^{7} \mathrm{M}_{\odot}$ at redshifts between 100 and 10 (Tegmark et al. 1997). Isolated simulations favoured halo masses of $\sim 7 \times 10^{5} \mathrm{M}_{\odot}$ (Fuller \& Couchman 2000), while cosmological simulations favoured $\sim 7 \times 10^{5} \mathrm{M}_{\odot}$ (Yoshida et al. 2003) and found no dependence on redshift.

Recent simulations continue to apply increasingly sophisticated models, including detailed radiative feedback, with Umemura et al. (2013) finding that multiple stars may form within haloes of mass $\sim 10^{5} \mathrm{M}_{\odot}$. Xu, Wise, \& Norman (2013) study Pop III star formation at high resolution over a relatively large volume, and find that Pop III stars form in haloes between $4 \times 10^{6} \mathrm{M}_{\odot}$ and $3 \times 10^{8} \mathrm{M}_{\odot}$, with the majority forming in haloes with masses of a few $\times 10^{7} \mathrm{M}_{\odot}$. In their simulations, Pop III stars inside more massive haloes are the result of mergers of small haloes, rather than forming in the more massive haloes themselves. We note that significantly more work has been done on the earliest stars that form in the largest over-densities at high redshift, that will be progenitors of massive galaxies, than in smaller over-densities that are progenitors of lower mass galaxies such as the LMC.
In our simulations, the first stars form within a halo with virial mass $\sim 5 \times 10^{7} \mathrm{M}_{\odot}$, at $z \sim 13$. Only a single star particle $\left(7 \times 10^{3} \mathrm{M}_{\odot}\right)$ that forms in this earliest collapsing halo has zero metallicity, and it is able to pollute the surrounding region such that subsequent star particles have some metals. Just to make it clear, our model is not capturing details of the progression of star formation and self-enrichment within these first star forming sites, which remains uncertain. Rather, our labels of zero metallicity stars reflect the properties of the most metal-poor stars formed in any given region. Thus, it is entirely possible that some fraction of the star particle forming in haloes that collapse from primordial gas will nevertheless contain metals that were inherited from other stars forming in the same temporally extended burst. For our purposes here then stars formed in such self-enriched primordial star clusters are included in the distribution that we have labelled as zero metallicity, or primordial stars. Similarly, if the first stars in LMC mass galaxies form in lower mass systems at higher redshift than what we resolve, then presumably these would be incorporated into these collapsing regions that we do resolve. Yet the evolutionary fate of these stars, whether they are primordial or simply low metallicity, is well modelled in our simulations.

The formation of primordial stars in our simulation then proceeds as independent proto-galactic regions collapse to $5-8 \times 10^{7} \mathrm{M}_{\odot}$. We have identified that 14 such regions that house primordial star formation exist, and that they collapse between redshift $\sim 13$ and redshift $\sim 3$. The number of star particles in each independent site of primordial star formation ranges from 1 to 3 , meaning a mass of (0.7-2.1 $\times$ $\left.10^{4} \mathrm{M}_{\odot}\right)$. Again, some uncertainty remains as to how star formation should proceed in each of these sites. In particular, our simulations show no dependence of the mass of stars formed in these sites with redshift. Thus, a caveat of our model is that the early self-pollution of the separate sites of primordial star formation will be relatively similar and in particular are independent of redshift. A strong redshift dependence may have an effect on the shape of the radial distribution of early generation stars, as shown in Section 4.

\section{RESULTS}

In Figure 1, we show the star formation history (SFH) for both the ensemble of stars within the virial radius (black) and the extremely metal-poor (EMP) population $\left(\left[\mathrm{Z} / \mathrm{Z}_{\odot}\right]<-4\right.$, in red). EMP stars are all older than $\sim 10$ Gyr and form in $4-5$ discrete 'bursts', while the overall star formation rate is fairly constant over final $\sim 10$ Gyr of the simulation (with a slight upturn in the final $\sim 300 \mathrm{Myr}){ }^{7}$

In Figure 2, we show the age-metallicity relation (AMR) of the ensemble population of stars in our LMC

\footnotetext{
${ }^{7}$ Figure 1 is somewhat analogous to Figure 1 of Pilkington et al. (2012b), although here, we sub-divide the ensemble and EMP populations, while in the latter, we only showed the star formation history of the analogous solar neighbourhood of $11 \mathrm{mChab}$ (blue curve, therein).
} 


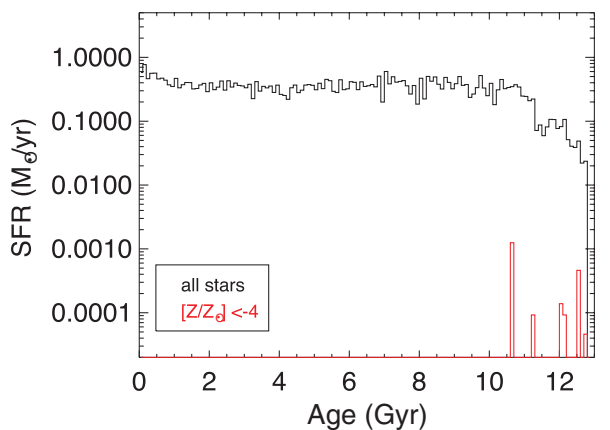

Figure 1. Star formation history of our LMC analogue, using all stars within the virial radius, colour-coded in black (for all stars) and red (extremely metal-poor stars: i.e., $\mathrm{Z}<\mathrm{Z}_{\odot} / 10000$ ). Here, the extremely metal-poor stars form in early, discrete, bursts, while the star formation history for the ensemble is fairly constant over the past $\sim 10 \mathrm{Gyr}$.

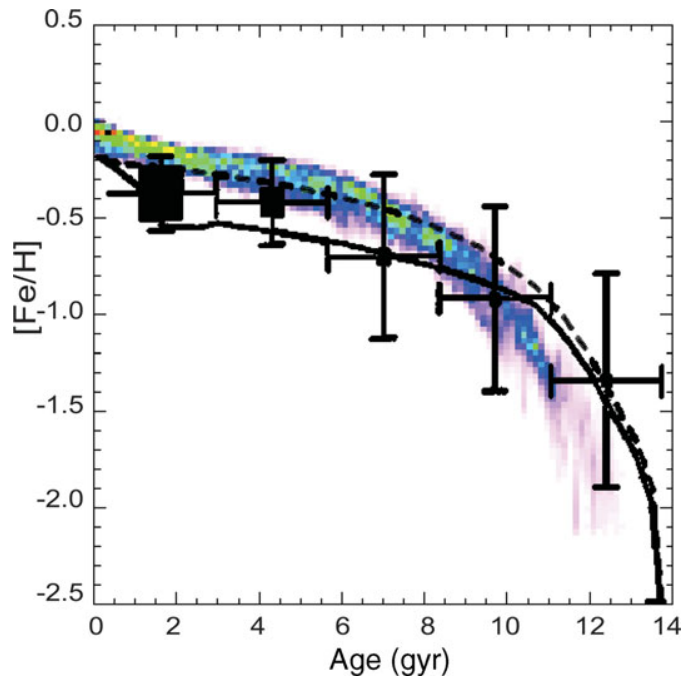

Figure 2. Age-metallicity relation for all stars within the virial radius of our simulated LMC analogue. The colour-coding of the contours is by density, ranging from purple (fewest stars) to green (most stars). The black curves and points represent analytical models and empirical data for the LMC, after Pagel \& Tautvaisiene (1998).

analogue. ${ }^{8}$ The overall trend (both qualitatively and quantitatively) is remarkably similar to that seen for the LMC, in nature (overlaid in Figure 2 with black symbols). The curves in Figure 2 correspond to the analytical models of Pagel \& Tautvaisiene (1998).

We next show the MDFs as a function of galactocentric radius, for the ensemble of stars in our LMC analogue (the black line within Figure 3). ${ }^{9}$ We note firstly that the stellar halo of the galaxy extends out to $\sim 40 \mathrm{kpc}$. The red line (radii $<5 \mathrm{kpc}$ ) shows that a low metallicity tail exists in the

\footnotetext{
${ }^{8}$ Figure 2 is analogous to the upper right panel of Pilkington et al.'s (2012b) Figure 2, albeit for the ensemble of stars rather than just the sub-set of 'solar neighbourhood' stars shown in the latter.

${ }^{9}$ The black curve of Figure 3 is analogous to the upper right panel of Pilkington et al.'s (2012b) Figure 3, albeit for the ensemble of stars rather than just the sub-set of 'solar neighbourhood' stars shown in the latter.
}

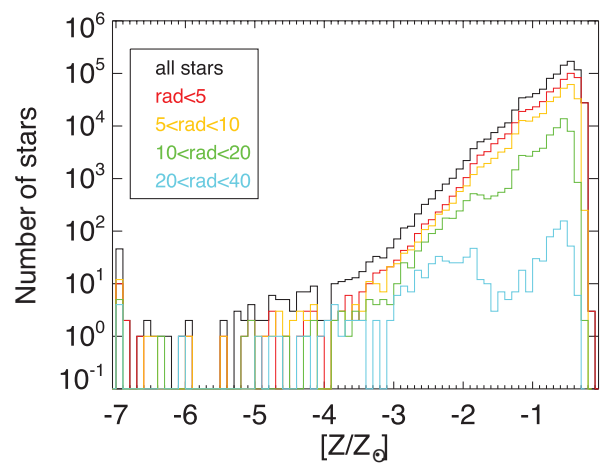

Figure 3. Metallicity distribution function of disc stars within our simulated LMC analogue, as a function of galactocentric radius: black (all stars), red (radii $<5 \mathrm{kpc})$, yellow $(5<$ radii $<10 \mathrm{kpc})$, green $(10<$ radii $<20 \mathrm{kpc})$, and cyan $(20<$ radii $<40 \mathrm{kpc})$.

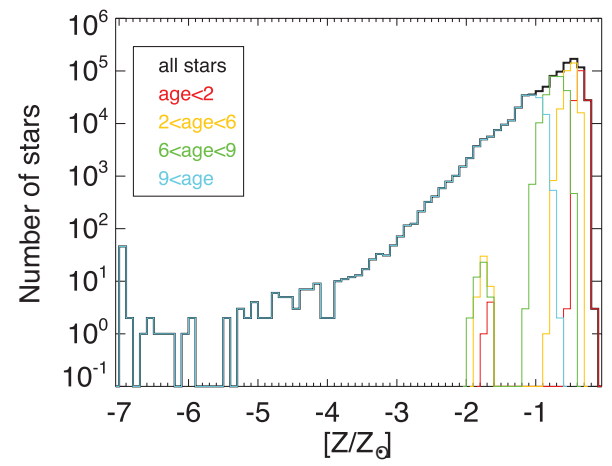

Figure 4. Metallicity distribution functions for the ensemble of stars within the virial radius of our simulated LMC analogue, sub-divided by stellar age. In black, all stars are shown; in red, only stars formed in the past $2 \mathrm{Gyr}$ are shown; yellow, green, and cyan correspond, respectively, to ages $2 \rightarrow 5 \mathrm{Gyr}$, $6 \rightarrow 9 \mathrm{Gyr}$, and $>9 \mathrm{Gyr}$.

main body of the galaxy, but that very few stars-fractionally speaking - have $\left[\mathrm{Z} / \mathrm{Z}_{\odot}\right]<-4$ (i.e., few EMPs). Conversely, as we move to larger radii, the fractionof EMPs increases (even if the overall numbers do not).

In Figure 4, the MDF is separated by stellar ages, with red being the youngest and cyan the oldest. Stars with $\left[\mathrm{Z} / \mathrm{Z}_{\odot}\right]<-2$ are older than $9 \mathrm{Gyr}$. The metallicity decreases with increasing age, as per the age-metallicity relation (Figure 2). The very youngest stars (red line, age $<2 \mathrm{Gyr}$ ) have a very narrow distribution at $\left[\mathrm{Z} / \mathrm{Z}_{\odot}\right] \sim-0.5$ and a few stars with $\left[\mathrm{Z} / \mathrm{Z}_{\odot}\right] \sim-2$. The oldest stars (light blue, ages $>9 \mathrm{Gyr}$ ) have a peak at $\left[\mathrm{Z} / \mathrm{Z}_{\odot}\right] \sim-1.0$, with a long tail. These trends are consistent with our earlier analysis of the 'bulge' and 'solar neighbourhood' regions of a comparable simulated dwarf (Figures 4 and 5 of Pilkington et al. 2012b are analogous to that of Figure 4 here, albeit the latter now shows the full ensemble of stars rather than just two spatially selected sub-sets employed in Pilkington et al.).

In Figure 5, we focus on the the zero metallicity (black) and EMP stars (red). We show the fraction of low metallicity 


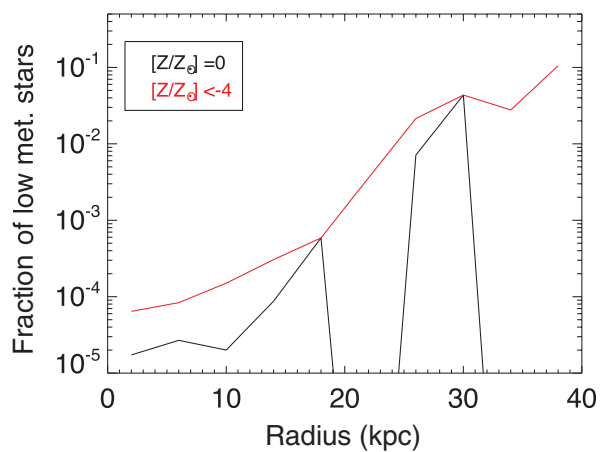

Figure 5. Fraction of zero metallicity (black) and EMP (red) stars (normalised by the total number of stars at each galactocentric radius).

stars as a function of galactocentric radius (normalised by the total number of stars in each radial bin). While the number statistics are low, as a fraction of the stellar population, low metallicity stars only become significant in the outer parts of the galaxy. We restate the caveat mentioned in Section 3 that the enrichment of the independent regions of primordial star formation is relatively uniform in our models, without a significant redshift dependence. Under this assumption, the predicted trends in Figure 5 will remain, although the absolute values of the fractions will vary according to the details of the earliest star formation within the earliest collapsing proto-galactic haloes.

We can now visualise the evolution of the galaxy and its metals, and identify the specific origin of the low metallicity stars. In Figure 6, time evolves from left to right, with the six columns corresponding to redshifts $z=5.5,4.2,3.1,2.0$, 1.0 , and 0 , respectively. We plot all baryons that form stars by $z=0$; each point can be a gas element, or it may have already formed a star. Thus, in the right-most column, only stars are shown because, by construction, it is redshift $z=0$; conversely, in the earlier time steps gas is also shown (which will later form stars), with the gas dominating increasingly to the left (i.e., at higher redshifts).

The colour distribution is the same for both rows in Figure 6: the highest metallicity is represented in blue, and as metallicity decreases the colour changes progressively to cyan, green, and yellow. In the top row, each baryon is coloured according to this mapping (i.e., to the metallicity of the star it will have formed by $z=0$ ). Thus, in the top-left panel, baryons that form the lowest metallicity stars (green, $\left[\mathrm{Z} / \mathrm{Z}_{\odot}\right]<-3$ ) are already collapsing in the densest regions. By contrast, the gas which will form stars with high metallicity (blue, $\left[\mathrm{Z} / \mathrm{Z}_{\odot}\right]>-1$ ) is in the outer, less dense regions at $z=5.5$, and will not form stars until later, by which time they are enriched.

In the bottom row of Figure 6, colours are assigned to the baryons by the metallicity they have at the time step of each panel. This shows that the enrichment is most evolved in the central region, where stars are forming, with the $z=$ 4.2 panel, in particular, showing that enrichment is quite inhomogeneous, with enrichment proceeding around locally collapsed regions. The region around the central main progenitor is the most enriched. Note that the order of overplotting the different metallicities is opposite in each row: in the top panels, the low metallicity baryons are overplotted on the ones with higher metallicity; in the bottom row, we follow the inverse process. In other words, the two panels of the far-right column are the same, but plotted in the inverse metallicity order.

The yellow diamonds in both panels of Figure 6 indicate baryons with zero metallicity (stars or the gas from which they form). Red diamonds are the extremely metalpoor $\left(\left[\mathrm{Z} / \mathrm{Z}_{\odot}\right]<-4\right)$ stars. Clearly, primordial gas is collapsing in several local regions and forming stars, prior to

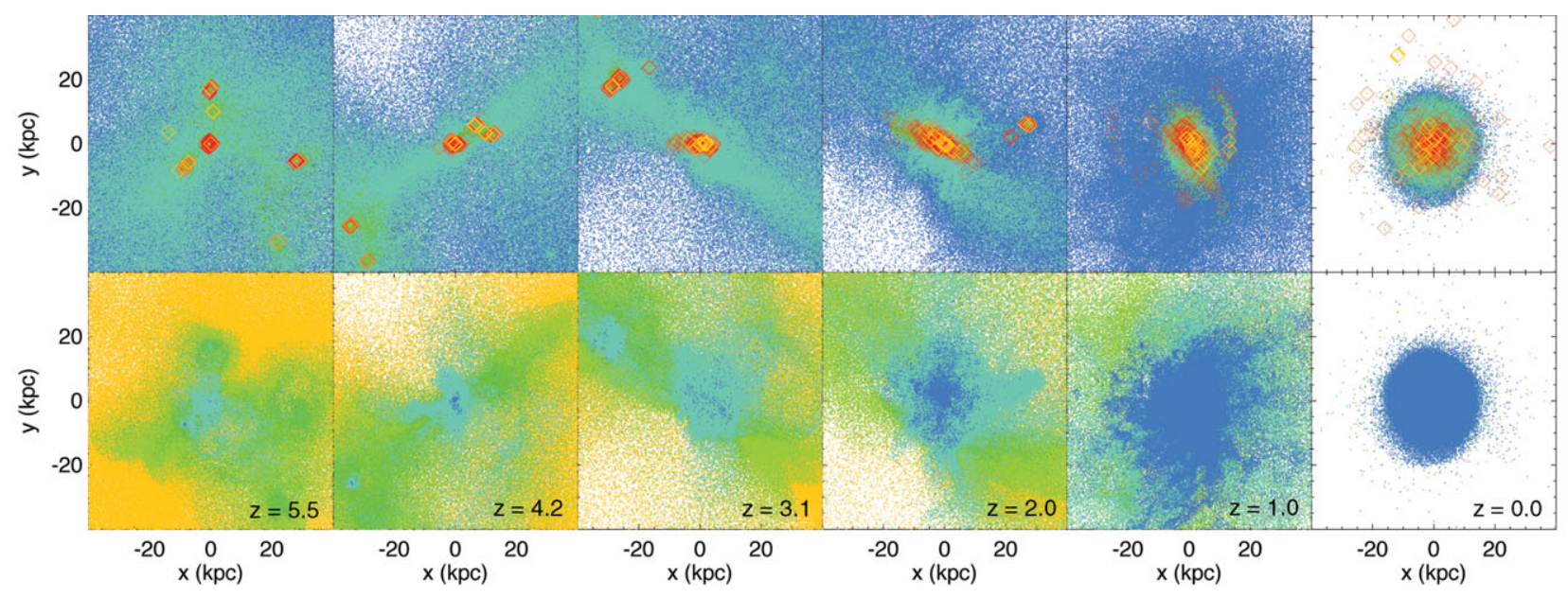

Figure 6. Temporal evolution of all baryons which will form stars by redshift $z=0$ in the $x-y$ plane with sides of $40 \mathrm{kpc}$. The columns, from left to right, correspond to redshifts $z=5.5,4.2,3.1,2.0,1.0$, and 0 , respectively. Baryons in the top row are coloured by the metallicity of the stars which they will form by $z=0$. Baryons in the bottom row are coloured according to the metallicity at each time step. The colour distribution is the same in both sequences: $\left[\mathrm{Z} / \mathrm{Z}_{\odot}\right]$ $>-1$ (blue), $-3<\left[\mathrm{Z} / \mathrm{Z}_{\odot}\right]<-1$ (cyan), $\left[\mathrm{Z} / \mathrm{Z}_{\odot}\right]<-3$ (green), and zero metallicity (yellow). In the top row, we have identified separately the baryons (i.e. stars and the gas from which stars will form) with $\left[\mathrm{Z} / \mathrm{Z}_{\odot}\right]<-4$ (yellow diamonds) and zero metallicty stars (red diamonds). 
accretion to the central region. This process is what results in the more extended distribution of the lowest metallicity stars, compared to the general population, which is dominated by stars that form at later times from gas that accretes to the central region before forming stars.

\section{CONCLUSIONS}

Cold dark matter cosmology is characterised by the hierarchical build-up of mass through merging processes. So long as stars are able to form within locally collapsing regions during an early rapid-collapse phase, they will subsequently be thrown into an extended distribution (a stellar halo) as the central galaxy is assembled. Using fully cosmological hydrodynamical zoom simulations of a dwarf galaxy, we have shown that we can expect this to occur in galaxies in the mass range of the LMC. In our simulation, the accreted stars extend out to $\sim 40 \mathrm{kpc}$ in a system which has double the stellar mass of the LMC.

We have further shown that we can expect that the first stars forming within high redshift, locally collapsing, regions, will form from primordial material, meaning that the first and second generation stars will have an extended distribution at $z=0$. This is in spite of the significant large scale supernova-driven outflows that occur in the simulated galaxies of our MaGICC program (Brook et al. 2012a, 2012b; Stinson et al. 2012). In our simulations, 14 independent sites of primordial star formation exist, as proto-haloes collapse to $\sim 5-8 \times 10^{7} \mathrm{M}_{\odot}$ between redshift $\sim 13-3$. Later star formation is dominated by in situ star formation in the inner region of the galaxy, meaning that it is in the outer regions where the largest fraction of first and second generation stars will be found at $z=0$. So long as the enrichment of the independent regions of primordial star formation is relatively uniform, without a significant redshift dependence, these predicted trends will remain, although the absolute values of the fractions will vary according to the details of the earliest star formation within the earliest collapsing proto-galactic haloes.

Although this comparison of a simulated galaxy using hydrodynamics within a cosmological framework has provided interesting insights into the expectations regarding the oldest populations in low mass galaxies such as the LMC, a larger statistical suite of simulations are required to make a firmer one-to-one comparison with the LMC-Milky Way system as a whole. We note that the extended population of stars in the LMC, as predicted in our simulations, evokes the predictions made by Bovill \& Ricotti 2011 of 'ghost halos' around isolated dwarfs as remnants of primordial star formation.

\section{ACKNOWLEDGEMENTS}

CBB is supported by the MICINN (Spain) through the grant AYA2009-12792. BKG acknowledges the support of the UKâ Science \& Technology Facilities Council (ST/J001341/1). The gener- ous allocation of resources from STFC's DiRAC Facility (COSMOS: Galactic Archaeology) is gratefully acknowledged. We also thank the PRACE-2IP project (FP7 RI-283493) for support, in addition to the University of Central Lancashire's High Performance Computing Facility.

\section{REFERENCES}

Besla, G., et al. 2007, ApJ, 668, 949

Bovill, M. S., \& Ricotti, M., 2011, ApJ, 741

Brook, C. B., Kawata, D., Scannapieco, E., Martel, H., \& Gibson, B. K. 2007, ApJ, 661, 10

Brook, C. B., et al. 2011, MNRAS, 415, 1051

Brook, C. B., Stinson, G., Gibson, B. K., Wadsley, J., \& Quinn, T. 2012a, MNRAS, 424, 1275

Brook, C. B., et al. 2012b, MNRAS, 426, 690

Bullock, J. S., Kravtsov, A. V., \& Weinberg, D. H. 2000, ApJ, 539, 517

Chabrier, G. 2003, ApJ, 586, L133

Cioni, M.-R. L. 2009, A\&A, 506, 1137

Cole, A. A., Tolstoy, E. Gallagher, III, J. S., \& Smecker-Hane, T. A. 2005, AJ, 129, 1465

Cole, A. A., et al. 2009, in IAU Symposium, Vol. 256, IAU Symposium, ed. J. T. Van Loon, \& J. M. Oliveira, Cambridge University Press, 263

Diemand, J., Madau, P., \& Moore, B. 2005, MNRAS, 364, 367

Feast, M. W., Abedigamba, O. P., \& Whitelock, P. A. 2010, MNRAS, 408, L76

Fuller, T. M., \& Couchman, H. M. P. 2000, ApJ, 544, 6

Gibson, B. K. 1994, JRASC, 88, 383

Gibson, B. K. 2000, MmSAI, 71, 693

Gibson, B. K., Pilkington, K., Brook, C. B., Stinson, G. S., \& Bailin, J. 2013, A\&A, 554A, 47G

Guo, Q., White, S., Li, C., \& Boylan-Kolchin, M. 2010, MNRAS, 404, 1111

Harris, J., \& Zaritsky, D. 2009, AJ, 138, 1243

Haschke, R., et al. 2012, AJ, 144, 88

Holtzman, J. A., et al. 1999, AJ, 118, 2262

House, E. L., et al. 2011, MNRAS, 415, 2652

Klypin, A., Kravtsov, A. V., Valenzuela, O., \& Prada, F. 1999, ApJ, 522,82

Macciò, A. V., Dutton, A. A., \& van den Bosch, F. C. 2008, MNRAS, 391, 1940

Moore, B., et al. 1999, ApJ, 524, L19

Moster, B. P., Naab, T., \& White, S. D. M. 2013, MNRAS, 428, 3121

Moster, B. P., et al. 2010, ApJ, 710, 903

Muñoz, R. R., et al. 2006, ApJ, 649, 201

Norris, J. E., et al. 2013, ApJ, 762, 25

Pagel, B. E. J., \& Tautvaisiene, G. 1998, MNRAS, 299, 535

Piatti, A. E., \& Geisler, D. 2013, AJ, 145, 17

Pilkington, K., et al. 2012a, A\&A, 540, A56

Pilkington, K., et al. 2012b, MNRAS, 425, 969

Purcell, C. W., Bullock, J. S., \& Zentner, A. R. 2007, ApJ, 666, 20

Rubele, S., Girardi, L., Kozhurina-Platais, V., Goudfrooij, P., \& Kerber, L. 2011, MNRAS, 414, 2204

Scannapieco, E., et al. 2006, ApJ, 653, 285 
Smecker-Hane, T. A., Cole, A. A. Gallagher, III, J. S., \& Stetson, P. B. 2002, ApJ, 566, 239

Stinson, G., et al. 2006, MNRAS, 373, 1074

Stinson, G. S., et al. 2009, MNRAS, 395, 1455

Stinson, G. S. 2010, MNRAS, 408, 812

Stinson, G. S., et al. 2012, MNRAS, 425, 1270

Stinson, G. S., et al. 2013, MNRAS, 428, 129

Tegmark, M., et al. 1997, ApJ, 474, 1

Umemura, M., Susa, H., Hasegawa, K., Suwa, T., \& Semelin, B. 2013, Prog. Theor. Exp. Pys., 01A306 van der Marel, R. P., Alves, D. R., Hardy, E., \& Suntzeff, N. B. 2002, AJ, 124, 2639

White, S. D. M., \& Springel, V. 2000, in The First Stars, ed. A. Weiss, T. G. Abel, \& V. Hill, Springer-Verlag, 327

Xu, H., Wise, J. H., \& Norman, M. L. 2013, ArXiv e-prints

Yoon, S.-J., \& Lee, Y.-W. 2002, Sci, 297, 578

Yoshida, N., Sokasian, A., Hernquist, L., \& Springel, V. 2003, ApJ, 598, 73

Zolotov, A., et al. 2009, ApJ, 702, 1058 